\title{
Un análisis del papel del uso de modos no textuales de representación del conocimiento científico en exposiciones de estudiantes de formación profesional
}

\author{
Carlos Emilio Reigosa Castro \\ Facultade de Formación do Profesorado e IES Lucus Augusti.Lugo. España.carlos.reigosa@usc.es \\ ORCID:bttps:/ / orcid.org/0000-0002-2137-1471 \\ Paloma Blanco Anaya \\ Facultade de Formación do Profesorado. Lugo.España.paloma.blanco@usc.es \\ ORCID: https:/ / orcid.org/0000-0002-3347-1103
}

[Recibido: 14 Enero 2020. Revisado: 3 Febrero 2020. Aceptado: 27 Febrero 2020]

\begin{abstract}
Resumen: Describimos una experiencia de investigación-acción en la que analizamos cómo estudiantes de formación profesional incorporan modos no textuales de representación del conocimiento científico en presentaciones que elaboran para ser expuestas ante sus compañeros. Estudiamos en esos modos no textuales de qué tipo son, su integración y su capacidad de resumir información y, al hallar aspectos a mejorar, profundizamos en ello mediante un estudio de caso. Encontramos que el uso de modos no textuales de representación de conocimiento contribuye a conseguir progresos relevantes en relación con la alfabetización científica en términos comunicativos referentes a expresar y compartir conocimientos.
\end{abstract}

Palabras clave: Modos no textuales de representación de conocimiento, alfabetización científica, acción comunicativa, investigación-acción, emancipación

An analysis of the role of students' use of non textual modes of scientific knowledge representation in presentations of vocational training students

\begin{abstract}
Here is described an action research experience in which we study how the participant vocational training students use non textual modes of scientific knowledge representation in the presentations that they elaborate in order to be exposed to their classmates. We study in those non textual modes their type, integration and power of summarize information, and, as we found aspects to improve, we deepen through a case study. We conclude that the use of non textual modes of scientific knowledge representation can allow relevant progresses related to scientific literacy in communicative terms referred to expressing and sharing knowledge.
\end{abstract}

Keywords: Non textual modes of knowledge representation, scientific literacy, communicative action, action research, emancipation

Para citar este artículo: Reigosa C., Blanco A. (2020) Un papel del uso de modos no textuales de representación del conocimiento científico en exposiciones de estudiantes de formación profesional. Revista Eureka sobre Enseñanza y Divulgación de las Ciencias 17(2), 2102. doi: 10.25267/Rev_Eureka_ensen_divulg_cienc.2020.v17.i2.2102

\section{Introducción}

Una de las principales aportaciones de la investigación en didáctica de las ciencias es la de buscar soluciones, entre otras, a los problemas asociados a las dificultades del alumnado ante la comprensión de conceptos y principios científicos, y que los puedan transferir a su vida cotidiana (Gil, Carrascosa y Martínez 2000). En ese sentido, como señala Lemke (1997), uno de los aspectos que más atención requiere es el uso del lenguaje de las ciencias, siendo una de las dimensiones más importantes para la comunicación del conocimiento científico. En este trabajo compartimos con otros estudios una visión en torno a la enseñanza de las ciencias que le asigna un rol central al lenguaje en general y también al papel de las características específicas del lenguaje de la ciencia (puede verse una revisión en Prain y Hand 2016). Como ya indicó Vygotski (1934), pensamos a través del lenguaje, que es una herramienta social que 
nos enmarca en una comunidad (Wertsch y Rupert 1991), ya que el lenguaje compartido es una de las dimensiones que define a las comunidades y, en la comunidad científica, como en otras, abarca un conjunto propio y variado de herramientas semióticas de creación de significados y conocimiento cuyo dominio va mucho más allá de la mera comprensión de un léxico (Lemke 1997). De este modo, para comprender el saber científico es imprescindible la apropiación de herramientas semióticas específicas de la ciencia.

Por esto, como McDermott y Hand (2013) señalan, dentro de la educación científica se le debe otorgar un valor primordial al desarrollo de la capacidad de compartir conocimiento científico, dentro de la cual son centrales las destrezas de lectura y escritura, incluyendo los variados modos de representación de conocimiento usados por los científicos. Por ello consideramos que son necesarias investigaciones que contribuyan al conocimiento sobre cómo los estudiantes usan modos no textuales de representación de conocimiento, como tratamos de hacer con este artículo.

\section{Uso de distintos modos de representación del conocimiento científico para la alfabetización científica}

Una alfabetización científica completa debe dar soporte, entre otras cosas, al dominio de las formas de construir, transferir y representar conocimientos de la ciencia, dentro de las cuales coincidimos con Moje (2007) en que hay que poner énfasis en los llamados modos múltiples de representación. Como Prain y Hand (2016) indican, los productos escritos de los científicos son multimodales, porque incluyen no solo párrafos de texto, sino también modos gráficos y matemáticos de representación de conocimiento, es decir, modos no textuales de representación, los cuales componen los sistemas simbólicos de la ciencia y, además, también incluyen elementos como tablas o listas, que se consideran una evolución con respecto a los párrafos de texto. La escritura multimodal es la forma de comunicación entre científicos (McDermott y Hand 2016) e incluso hay autores que consideran que la ciencia tal y como la conocemos hoy en día comenzó a existir cuando se empezaron a usar formas no textuales para representar los objetos de la indagación científica (Cunningham 1988). En este trabajo partimos de la consideración de que los estudiantes deben tener oportunidades para usar los múltiples modos de representación de conocimiento científico distintos de párrafos de texto, acercándolos a la importancia de su uso y comprensión en la comunicación del conocimiento científico (Perales, 2006).

Dentro de la construcción de conocimiento científico, es central el movimiento entre diferentes modos de representarlo, ya que los mismos conceptos pueden representarse con distintos formatos (Lundsford, Melear, Roth, Perkins y Hickok 2007). De esta manera, usar distintos modos de representación implica la adquisición de destrezas en el manejo de expresiones textuales, gráficas y matemáticas pero no de forma independiente, sino integrada, sinérgica, y en conexión con sus referentes reales en el mundo físico, aunque como Lundsford et al. (2007) apuntan, no todos son equivalentes, ya que los más potentes son los que resumen más información.

Un aspecto terminológico a aclarar es que, en la bibliografía también está ampliamente extendido el término inscripción, que designa todos los materiales no textuales que aparecen en los trabajos científicos (Latour y Woolgar 1995), y que facilitan la representación abstracta del mundo físico. Para homogeneizar la nomenclatura usada en este trabajo, optamos por el término modos de representación frente a inscripción. Aunque los dos nos parecen válidos, pensamos que modos de representación evoca en mayor grado el conocimiento representado. 


\section{La alfabetización científica desde una perspectiva emancipadora}

Un aspecto a tener en cuenta en este estudio es que el alumnado participante son adultos, en concreto estudiantes de formación profesional, a diferencia de lo más habitual en las investigaciones educativas, lo cual tiene implicaciones específicas, ya que la educación de adultos tiene un papel social integrador fundamental y un carácter diferenciado (Sárrate y Pérez 2005): su carácter voluntario, el papel de la experiencia, la autonomía del alumnado.... Para fomentar que estos estudiantes generen actitudes positivas en torno a la participación en cuestiones científicas, resulta oportuno que avancen en la comprensión y manejo de las herramientas discursivas de la ciencia. A este respecto, tendremos en cuenta la propuesta de Salvá y Latorre (1998), quienes se muestran a favor de optar por un enfoque comunicativo con base en la teoría crítica (Habermas 1987), útil para fomentar el diálogo para expresar conclusiones, para la toma de decisiones o para el establecimiento de normas.

Concretando la educación de adultos en lo relativo a la alfabetización científica, de Arriba (2007) propone un planteamiento holístico y recuerda la importancia de la adquisición de competencias básicas, como son las relacionadas con el discurso. Para esto, una posibilidad es articular propuestas educativas en torno a la realización por parte de los estudiantes de exposiciones ante sus compañeros, como se hará en la intervención aquí analizada, lo cual puede facilitar el desarrollo de la capacidad de movilizar y compartir conocimientos al tiempo que se interiorizan elementos propios de la cultura científica, por ejemplo incorporando en su discurso modos no textuales de representación de conocimiento. Consideramos que solicitar al alumnado que elabore presentaciones sobre temas científicos y las exponga a sus compañeros es una estrategia para ayudarles a abrir cauces de participación en el discurso científico. Por ello, pensamos que resulta conveniente documentar las dificultades de su elaboración en el aula, como se pretende hacer aquí. Esto puede complementar otros trabajos que analizan la capacidad de resumir información de modos no textuales de representación de conocimiento usados por los estudiantes en informes de laboratorio (Reigosa 2010) o en la comunicación de noticias científicas (Gebre y Polman 2016).

La participación en la comunicación científica socialmente significativa lleva implícita la búsqueda de entendimiento o inteligibilidad, lo que se relaciona con lo que Habermas (1987) llama acción comunicativa, orientada a la interpretación mutua y al entendimiento. Este autor opone la acción comunicativa a la integración sistémica de las acciones, en la que los sujetos se relacionan con su entorno exclusivamente desde el punto de vista de lograr el éxito particular. $\mathrm{Si}$ se pretende que la enseñanza de las ciencias contribuya a fomentar un verdadero empoderamiento del alumnado, esto no pasa por ayudarles simplemente a conseguir sus fines o el éxito escolar o a interiorizar destrezas o estrategias útiles para la progresión social, sino por ofrecerles contextos en los que se les den oportunidades para coordinar sus acciones en base al entendimiento y a la comprensión mutua usando el lenguaje de las ciencias, lo cual es acertado también con alumnado adulto. Una perspectiva del currículum científico escolar orientada al entendimiento está en relación con una visión democrática de la sociedad basada en la inteligibilidad y el debate abierto, y es coherente con un enfoque educativo dirigido sobre todo a la mejora de la sociedad y no exclusivamente al avance personal de aquellos estudiantes que alcancen logros y títulos académicos.

Si entendemos la alfabetización científica como la capacidad de implicarse en temas de ciencias, usando ideas científicas como ciudadanos reflexivos, como se propone en el programa PISA (OCDE 2016), experiencias como la que aquí se muestra pueden ser útiles para favorecer una apropiación del lenguaje de la ciencia que contribuya a comprender el mundo real y actuar en él. Para ello la apropiación de las formas de creación de significados propias de la comunidad científica se hace necesaria para favorecer la emancipación de las 
personas, ayudando a los estudiantes a comprender de forma situada los fundamentos del conocimiento científico, el cual de esta manera no se les presenta como un saber fuera del alcance del ciudadano medio.

\section{Objetivos de investigación}

Por todo lo expuesto, este trabajo se realizó con el interés de indagar acerca del uso de modos no textuales de representación de conocimiento científico. El contexto era un grupo de estudiantes de formación profesional y el estudio se concretó en los siguientes objetivos de investigación:

1- Analizar la tipología, el grado de integración y la capacidad de resumir información de los modos no textuales de representación de conocimiento científico usados por los participantes.

2- Examinar la percepción de los asistentes sobre el uso de esos modos no textuales de representación y su comprensión de los contenidos expuestos.

\section{Metodología}

La metodología fue de carácter cualitativo, pretendiendo el desarrollo de interpretaciones que nos sirvan para entender con mayor profundidad el proceso educativo estudiado y estableciendo comparaciones entre distintas situaciones (Yin, 2003). En relación con la noción de generalizabilidad analítica (Kvale 1996), el objetivo era elaborar interpretaciones, constructos y teorías útiles para comprender mejor otras situaciones y para intentar mejorarlas. En este enfoque, son habituales metodologías cualitativas y descripciones detalladas de los contextos, lo cual aquí complementamos con análisis estadísticos de datos cuando resulta pertinente, usando técnicas no paramétricas, en concreto, tests $\chi^{2}$ y Kruskal-Wallis.

\section{Participantes, contexto e intervención}

La experiencia se llevó a cabo en un instituto público urbano de España con alumnado del $2^{\circ}$ curso del ciclo formativo de formación profesional (FP) de grado superior Laboratorio de Análisis y de Control de Calidad con una muestra de nueve participantes cuyas edades oscilaban entre 21 y 38 años. El profesor era uno de los autores de este artículo. Consideramos necesario indicar que, aunque es posible que el trabajo con alumnado de FP no sea el contexto más frecuente para el desarrollo de estudios acerca de aspectos relacionados con la enseñanzaaprendizaje de las ciencias, los técnicos de algunas ramas de FP, como la Química, van a desarrollar su vida laboral en contextos en los que se practica la ciencia y, por tanto, deben introducirse en su cultura.

El planteamiento de la intervención se realizó en términos de investigación-acción emancipadora (Kemmis y McTaggart 1988), buscando facilitar el empoderamiento del alumnado mediante el progreso en el manejo del lenguaje de la ciencia. De acuerdo con McKernan (1999), el objetivo era doble: por un lado, intrínseco de mejora de la situación social analizada, para así beneficiar al alumnado participante, al profesor y a la sociedad en general, y, por otro, extrínseco de creación de significados útiles para la comunidad educativa.

El módulo (asignatura) en el que se desarrolló la experiencia, Calidad y Seguridad en el Laboratorio, duró de septiembre a marzo. Entre abril y junio, los estudiantes que superaron todos los módulos realizaron prácticas formativas en centros de trabajo. El alumnado que no superó alguno recibió clases de recuperación en esos meses. Desde principios de curso, los estudiantes tenían que elaborar productos en los que debían incluir no solo párrafos de texto sino también otros modos de representación tales como tablas, ecuaciones, diagramas de flujo, histogramas de frecuencias, gráficas relacionando distintas propiedades físico-químicas, 
imágenes... Los estudiantes habían recibido formación sobre la elaboración de esos elementos y se había evaluado si los confeccionaban correctamente.

En el segundo trimestre, se les pidió que, en parejas (excepto un grupo de tres), elaborasen una presentación con una herramienta informática y la expusiesen. Así, conformaron cuatro grupos (A, B, C y D). La presentación debía versar sobre un apartado relacionado con los riesgos y normas de seguridad en los laboratorios, que cada grupo escogió de una lista. Consideramos que esta tarea permite a los estudiantes participar en prácticas de la cultura científica tales como consultar, interpretar e implementar prácticas seguras de trabajo en el laboratorio,

Esta era la primera vez que, en este módulo, elaboraban y exponían una presentación. Los estudiantes recibieron indicaciones sobre el formato: extensión máxima, tamaño de letra... Sin embargo, no se les dieron pautas acerca de la inclusión de modos no textuales de representación. Después, aunque podían plantear preguntas al profesor, debían ser ellos y ellas quienes tomaran las decisiones durante el proceso. Como explicaremos en el apartado de resultados, durante esta fase y después, cuando los estudiantes expusieron sus presentaciones, hallamos aspectos que consideramos susceptibles de mejora. Por ello se decidió realizar un análisis posterior mediante un estudio de caso con la única alumna que suspendió en marzo y que, por tanto, asistió a clases de recuperación entre abril y junio. A esta alumna, que pertenecía al grupo A, se le pidió que elaborase otra exposición para presentarla ante sus compañeros y se documentó el proceso con los documentos elaborados por ella y con información acerca de su percepción, la del profesor y la de otros observadores.

Esta estudiante, Yésica (pseudónimo), migró a España desde otro país en 2012 y tuvo un hijo en diciembre del curso en el que se desarrolló está experiencia, lo cual tuvo una importancia significativa para que no superase el módulo en marzo, a pesar de que se le dieron actividades de refuerzo.

Los contenidos en torno a los cuales Yésica elaboró la nueva presentación se englobaban dentro de un bloque relativo a la quimiometría. En concreto, debía elaborar y exponer una presentación explicando qué es un dendrograma y cómo se elabora. Los dendrogramas (Ramis y García 2001) son una herramienta ampliamente usada en química analítica y en otros campos. Permiten agrupar objetos en base a los valores medidos de varias variables, estableciendo el nivel de la semejanza de dichos objetos. Escogemos este tema para la segunda parte del estudio por su complejidad conceptual y matemática, que implica integrar de forma significativa conocimientos de química y estadística, lo cual pensábamos que podía propiciar mayor complejidad en el uso de modos no textuales de representación de conocimiento.

Yésica recibió formación sobre los dendrogramas, que fueron introducidos al tiempo que los empleaba para analizar supuestos prácticos. A continuación elaboró la presentación, la cual fue revisada y comentada por el profesor antes de realizar dos exposiciones con dos grupos diferentes de estudiantes: uno de $1^{\circ}$ curso de su ciclo formativo y otro que eran sus compañeros que habían aprobado en marzo.

\section{Toma y análisis de datos}

Para dar respuesta al primer objetivo de la investigación (analizar la tipología, el grado de integración y la capacidad de resumir información de los modos no textuales de representación de conocimiento científico usados), los datos recogidos consistieron en las presentaciones elaboradas primero por cada grupo y después por Yésica en solitario. Estas presentaciones fueron analizadas empleando las rúbricas de los tres apartados de la tabla 1, adaptadas a partir de McDermott y Hand (2016) y Lundsford et al. (2007). 
Tabla 1. Complejidad de los modos no textuales de representación

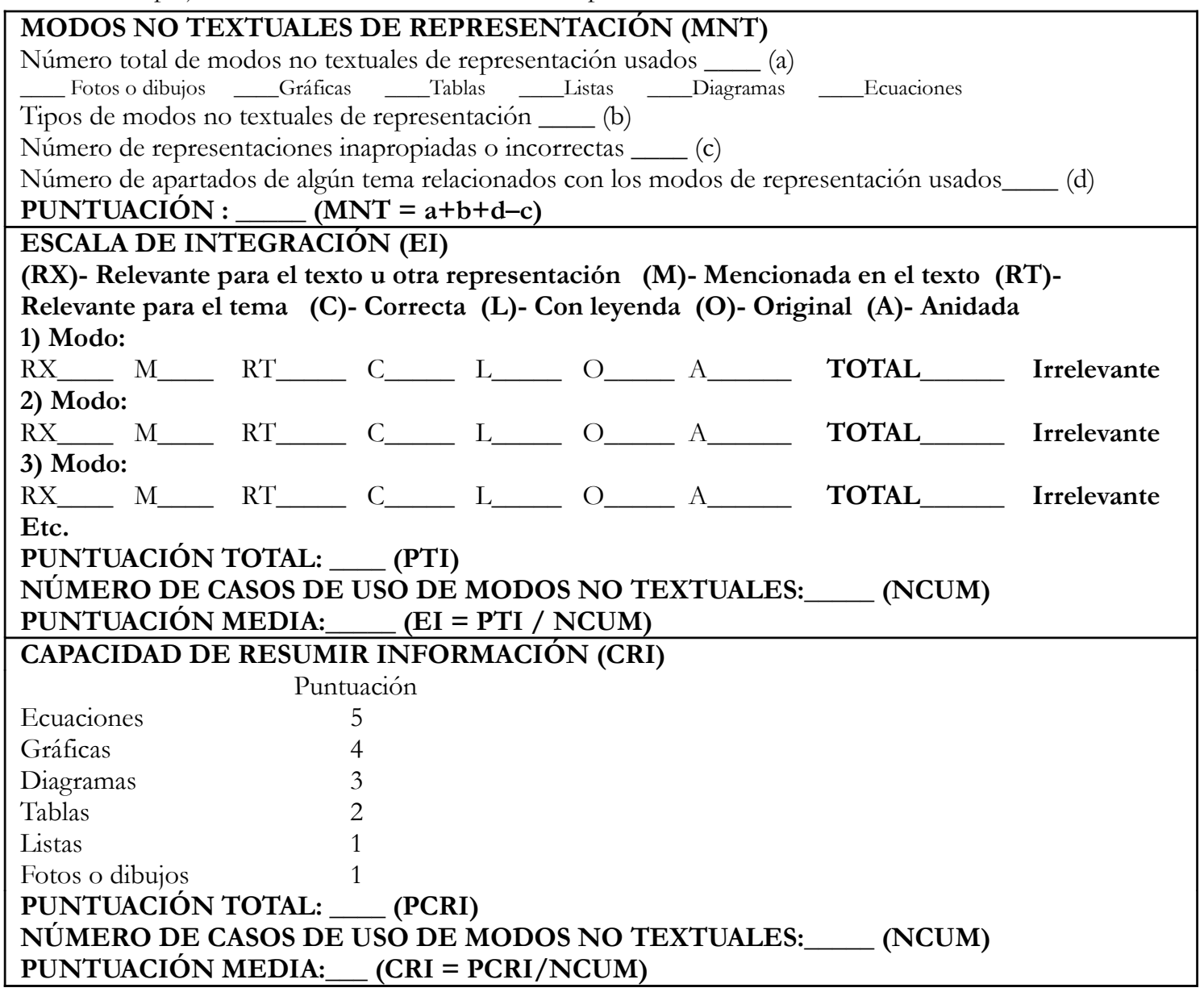

En primer lugar, el apartado Modos No Textuales de Representación (MNT), tomado del trabajo de McDermott y Hand (2016), valora el número de modos no textuales de representación usados y el número de temas que representan. Podría haber otros modos no textuales no indicados ahí, como vídeos por ejemplo, pero no fueron usados por los participantes. El parámetro (a) recoge la cantidad total de modos no textuales de representación usados, mientras que el (b) puntúa la variedad de modos no textuales. El parámetro (c) penaliza la introducción de representaciones inapropiadas o incorrectas y el (d) se refiere a la conexión de esos modos no textuales con temas o apartados de los contenidos relacionados. Es conveniente aclarar que aquí, y en todo el estudio, nos referimos a representaciones externas, plasmadas en productos elaborados por los estudiantes, y no a representaciones mentales.

El siguiente apartado de la tabla 1, Escala de Integración (EI), pretende ver el grado de integración de los distintos modos no textuales de representación. Para cada caso de uso de un modo de representación se comprobó si se daban una serie de características indicadas en la tabla 1 ("Correcta", "Con leyenda", etc.) que muestran el grado en que dicho uso de un modo de representación está integrado en el producto elaborado por los estudiantes. Este apartado está basado también en el trabajo de McDermott y Hand (2016), pero las características propuestas por estos autores han sido modificadas sustancialmente para adaptarlas a las presentaciones elaboradas por los participantes en este estudio. Hemos realizado la inclusión de: 
1) El ítem "original" $(\mathrm{O})$, haciendo referencia a modos no textuales de representación elaborados por ellos y no copiados y pegados de otra fuente.

2) La característica "anidada" (A), para indicar modos no textuales de representación introducidos en otros (por ejemplo, una foto introducida en una tabla), lo cual indica un uso versátil, significativo, intencional e integrado de los mismos. Hemos llamado modo no textual de representación anidado al que se inserta en otro, al que hemos denominado anfitrión.

3) La casilla "irrelevante", para los casos que no eran pertinentes.

A cada una de esas características del apartado EI se dio un punto, los cuales fueron sumados según se explica en la tabla 1.

Finalmente se valoró la potencia de los modos no textuales de representación empleados, entendida como su capacidad de resumir información (CRI), escala adaptada a partir de Lundsford et al. (2007). Con respecto a las categorías usadas por esos autores, hemos realizado modificaciones para que coincidan con las del apartado MNT.

En definitiva, hemos tenido que realizar cambios sustanciales en las rúbricas de McDermott y Hand y Lundsford et al. para llegar a las de la tabla 1 (sobre todo en los apartados EI y CRI) con el fin de llegar a disponer de una herramienta que se adapte plenamente a los productos de los estudiantes participantes y cuyos apartados sean coherentes y armónicos entre sí. La aplicación de las rúbricas de la tabla 1 en el análisis de los datos fue realizada independientemente por los dos autores del trabajo para aumentar la intersubjetividad de la codificación de los datos (Flick 2004). Posteriormente se hizo una puesta en común en la que los casos en los que había desacuerdo fueron resueltos de forma negociada.

Además, para profundizar en el uso por parte de los estudiantes de modos no textuales de representación de conocimiento científico, consideramos conveniente no sólo describirlo, sino además indagar acerca de la percepción del alumnado en lo referente a su manejo, como se indica en el $2^{\circ}$ objetivo de este estudio. Para obtener información al respecto, en la segunda fase del estudio se le pidió a Yésica que redactase diversos ensayos breves a lo largo del proceso de elaboración de su presentación. Simultáneamente, el profesor cumplimentó un registro anecdótico (McKernan 1999), optando por un formato no estructurado para recoger todo tipo de datos o acontecimientos juzgados subjetivamente como relevantes.

En los datos recogidos se buscaron indicios que denotasen en la percepción de Yésica interés (o la falta del mismo) por el uso de modos no textuales de representación de conocimiento. Se valoraron los siguientes aspectos relacionados con la motivación (Hulleman y Harackiewicz 2009): 1) Expectativas de éxito escolar, 2) interés intrínseco por el tema, 3) utilidad, 4) desarrollo de destreza, y 5) planes futuros. Además de esos aspectos, valoramos el peso que para ella tenía buscar el entendimiento por parte de sus compañeros, en relación con la noción ya discutida de acción comunicativa (Habermas 1987).

También examinamos, tanto la percepción de los asistentes a las exposiciones de Yésica (30 compañeros y compañeras del ciclo formativo) sobre el uso de los modos no textuales de representación, como su comprensión de los contenidos expuestos. A estos asistentes se les pidió que respondiesen individualmente por escrito a las preguntas de la tabla 2. Incluir en el análisis la visión de los estudiantes permite progresar en la comprensión del caso analizado, yendo más allá de la triangulación de observadores para llegar a una cuadrangulación (McKernan 1999) con cuatro puntos de vista: los del investigador-actor, la investigadora externa, la alumna participante y otros estudiantes. Dar cancha a la visión del alumnado favorece una relación horizontal docente-estudiantes e implica una autoimagen del profesorado como agente reflexivo facilitador del aprendizaje (Vázquez, Jiménez y Mellado 2019) y como intelectual transformativo (Giroux 1990). 
Tabla 2. Preguntas hechas a los estudiantes que asistieron a la exposición

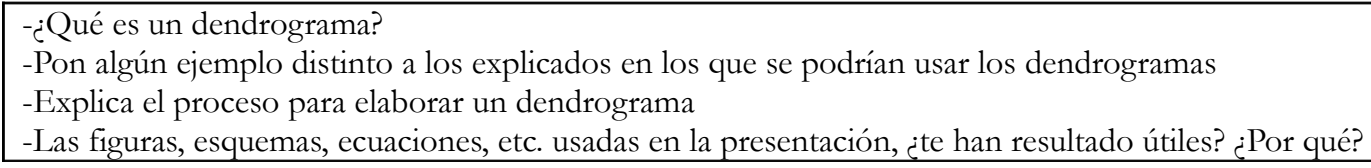

El análisis de estos datos se realizó de modo inductivo, buscando que la teoría emergiera en interacción con los datos (Glaser y Strauss 1967), aunque mediada por el planteamiento del análisis. La plausibilidad de los constructos e interpretaciones desarrolladas fue revisada cíclicamente por ambos autores.

\section{Resultados y discusión}

Los resultados se dividen en tres subapartados. En el primero se comentan los resultados referidos al uso de modos no textuales de representación en las presentaciones elaboradas por todos los grupos, en el segundo los resultados que derivan del mismo análisis pero centrándonos en la presentación elaborada por Yésica en solitario y, en el tercero, la información obtenida a partir del análisis de la percepción de Yésica, del profesor y de los asistentes a las exposiciones.

\section{Análisis de las presentaciones grupales}

En esta fase de la investigación se aplicaron las rúbricas de la tabla 1 a las presentaciones sobre seguridad en los laboratorios elaboradas por el total de grupos. En total se analizaron tres dimensiones (tipo, integración y potencia) de 158 modos no textuales de representación de conocimiento científico.

Los resultados referentes al apartado MNT de la tabla 1 se muestran en la figura 1 y en la figura 2 se detallan los parámetros a, b, c y d de este apartado.

Puede observarse en la figura 1 que el número total de modos no textuales de representación de conocimiento científico empleados por los cuatro grupos son distintos (76-38-23-21, respectivamente), siendo las diferencias estadísticamente significativas $\left(\chi^{2}=49.3, p=0.000\right)$. Igualmente, las puntuaciones finales del parámetro MNT (figura 2) tienen diferencias notables entre los grupos (95-53-39-35) y hay un escaso número de uso de modos no textuales que no dan información y que hemos considerado inapropiados (4-3-1-0).

Aparte de las diferencias entre los grupos en cuanto al número total de modos no textuales de representación, también las hay en cuanto al tipo de modos no textuales empleados. Las características más diferenciadoras de cada grupo son:

- Grupo A: predominio de fotos y dibujos. La gran abundancia de estos elementos en este grupo es lo que explica su mayor número total de modos no textuales de representación con respecto a otros grupos, lo cual es a su vez la causa de su mayor valor para el parámetro MNT.

- Grupo B: predominio de fotos y dibujos pero también de listas.

- Grupo C: uso variado y equilibrado de distintos modos no textuales de representación.

- Grupo D: ausencia de fotos y dibujos pero uso importante de listas. 


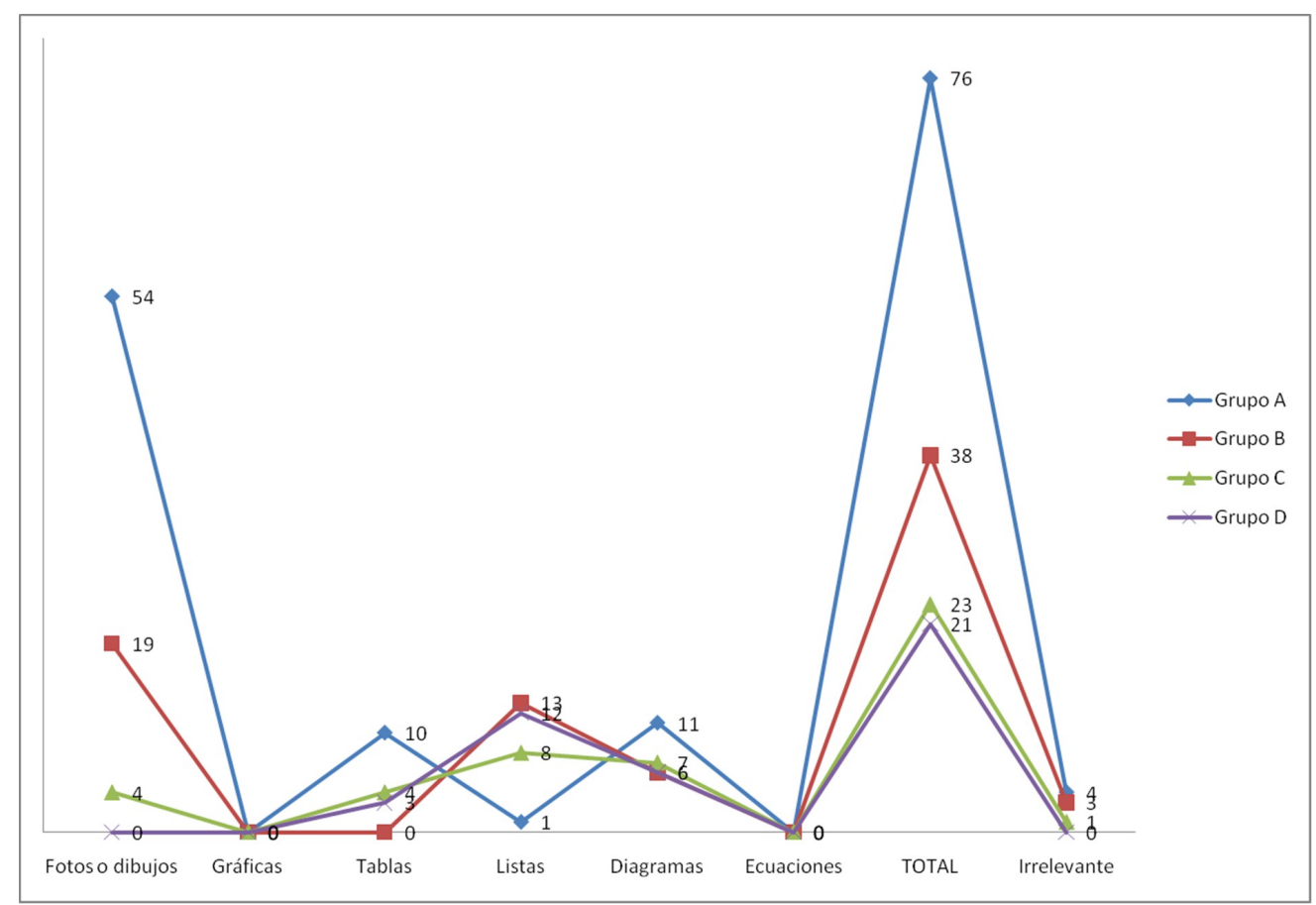

Figura 1. Modos no textuales de representación (MNT) usados por los estudiantes.

Una semejanza entre los cuatro grupos es la ausencia de gráficas y ecuaciones. Otro aspecto común es el importante número de apartados conceptuales introducidos en las presentaciones (apartado d, figura 2), para lo cual no hay diferencias significativas entre los grupos $\left(\chi^{2}=2.41, \mathrm{p}=0.491\right)$. Pero, a pesar de existir esas coincidencias, consideramos que los cuatro grupos tienen cuatro estilos claramente diferenciados en el uso de modos no textuales de representación en sus presentaciones, lo que indica distintas percepciones acerca de cómo debe ser una presentación y de cómo esta sirve al fin de hacer una buena exposición.

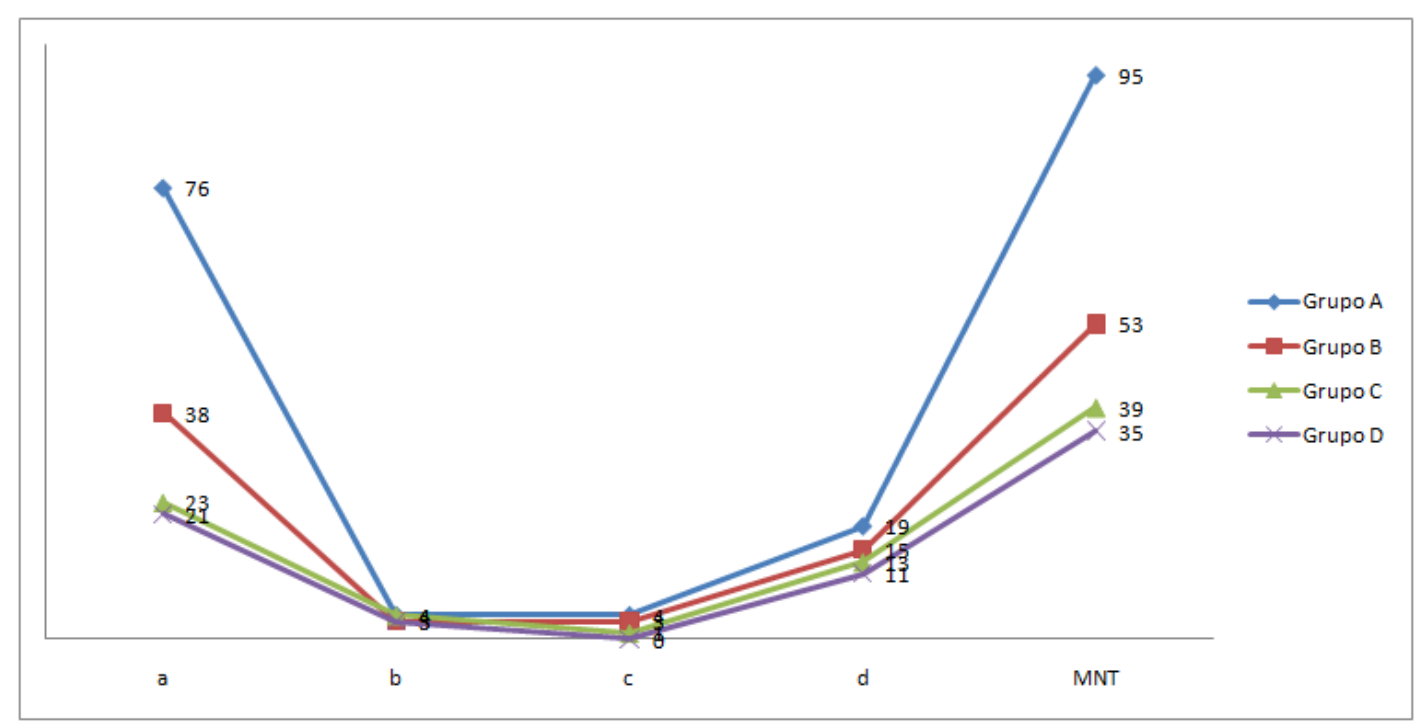

Figura 2. Parámetros del apartado MNT para los productos de los estudiantes (a = número total de modos no textuales; $\mathrm{b}=$ tipos de modos no textuales; $\mathrm{c}=$ número de representaciones inapropiadas; $\mathrm{d}=$ número de apartados relacionados con las representaciones).

En cuanto al siguiente apartado de la tabla 1, EI, referente a la integración de los modos no textuales de representación, los resultados se muestran en la tabla 3. Un test de Kruskal-Wallis indica diferencias significativas entre los valores de $\mathrm{EI}(\mathrm{H}=15.0, \mathrm{p}=0.002)$. Se puede ver que el grupo D, que era el que obtenía un valor más bajo para MNT, es el que obtiene mayor EI: el 
grupo que usa menos modos no textuales de representación es el que los tiene más integrados en las presentaciones, por lo que inferimos que sólo usa los modos no textuales que considera más necesarios.

Tabla 3. Valores obtenidos en el apartado EI (Escala de Integración)

\begin{tabular}{|cccc|}
\hline Grupo & PTI & NCUM & EI \\
\hline A & 334 & 76 & $\mathbf{4 , 3 9}$ \\
B & 157 & 38 & $\mathbf{4 , 1 3}$ \\
C & 100 & 23 & $\mathbf{4 , 3 5}$ \\
D & 108 & 21 & $\mathbf{5 , 1 4}$ \\
\hline
\end{tabular}

Dentro de las características que contribuyen a la puntuación del apartado EI, pensamos que merece especial atención qué modos no textuales están anidados y cuáles son originales (tabla 4).

En el grupo A hay un gran porcentaje de anidamiento, pero, sin embargo, presenta un bajo porcentaje de originalidad, debido a que este plantearon su presentación sobre todo en base a imágenes descargadas de Internet, las cuales insertaban a menudo en tablas (adquiriendo la característica de anidadas).

Tabla 4. Modos no textuales de representación anidados y originales.

\begin{tabular}{|cccc|}
\hline Grupo & Anidado & Original & Total \\
\hline A & $39(51 \%)$ & $15(20 \%)$ & 76 \\
B & 0 & $13(34 \%)$ & 38 \\
C & $5(22 \%)$ & $13(56 \%)$ & 23 \\
D & $3(14 \%)$ & $21(100 \%)$ & 21 \\
\hline
\end{tabular}

Los otros grupos tienen un menor anidamiento pero mayor originalidad. En este sentido, resultan equilibradas las propuestas de los grupos $\mathrm{C}$ y $\mathrm{D}$, con un porcentaje moderado de anidamiento y, al tiempo, una originalidad alta.

En la tabla 5 se señalan los tipos de anidamiento encontrados. Había modos no textuales anfitrión que incluían más de uno anidado. Puede verse que lo más usual son tablas que incluyen fotos o dibujos y diagramas.

Tabla 5. Tipos de anidamiento.

\begin{tabular}{|c|c|}
\hline Modo anfitrión & Modo anidado \\
\hline Tabla (12) & $\begin{array}{c}\text { Foto o dibujo (28) } \\
\text { Diagrama (16) }\end{array}$ \\
\hline Lista (2) & Tabla (1) \\
Lista (1)
\end{tabular}

Por último, en cuanto al apartado CRI, los resultados se muestran en la tabla 6.

Tabla 6. Valores obtenidos en el apartado CRI (Capacidad de Resumir Información).

\begin{tabular}{|cccc|}
\hline Grupo & PCRI & NCUM & CRI \\
\hline A & 108 & 76 & $\mathbf{1 , 4 2}$ \\
B & 50 & 38 & $\mathbf{1 , 3 2}$ \\
C & 41 & 23 & $\mathbf{1 , 7 8}$ \\
D & 36 & 21 & $\mathbf{1 , 7 1}$ \\
\hline
\end{tabular}

Un test de Kruskal-Wallis da como resultado que, por escaso margen, hay diferencias significativas $(\mathrm{H}=8.19, \mathrm{p}=0.042)$ en la capacidad de resumir información que presentan los diferentes grupos. Es relevante que la potencia de los modos no textuales de representación de los grupos es más bien baja, ya que la media general es 1,49 y el valor máximo es 5 . Esto se debe al nulo uso de gráficas y ecuaciones, que son los modos no textuales de representación con mayor potencia. 
Considerando globalmente la información que nos proporciona la aplicación de las rúbricas de la tabla 1, podemos ver que hay un extenso uso de modos no textuales de representación en los productos elaborados por los estudiantes (de hecho, no había párrafos de texto en sus presentaciones), si bien cabe preguntarse cuál es la calidad de esos modos no textuales de representación.

En primer lugar, el número de casos en los que los modos no textuales de representación no dan información y que hemos considerado irrelevantes es muy bajo (8 de 158), lo cual indica un escaso uso de esos elementos como relleno u ornamentación vacua de las presentaciones. A mayores se ha comprobado que el alumnado introduce un número significativo de apartados conceptuales en sus presentaciones y que hay bastantes casos de uso de modos no textuales de representación anidados, lo cual se relaciona con un uso significativo e integrado de los mismos. Pero, sin embargo, el análisis también ha revelado aspectos mejorables:

- Predominio en algunos grupos del uso de fotos o dibujos descargados de Internet. El grupo que incluye más fotos o dibujos es el que tiene menor porcentaje de originalidad.

- La capacidad de resumir información de los modos no textuales de representación usados es pobre, lo cual se debe a la ausencia de los modos no textuales que resumen más información (gráficas y ecuaciones) y al uso extensivo de los que menos resumen (fotos o dibujos y listas).

- La integración de los modos no textuales de representación es más satisfactoria que la capacidad de resumir información, si bien también es susceptible de mejora.

Cabe indicar que los tres indicadores (MNT, EI y CRI) parecen ser independientes, ya que hay grupos que obtienen una puntuación alta en uno y baja en otro. Eso nos permite decir que los apartados de la tabla 1 nos proporcionan información sobre dimensiones distintas, las cuales necesitan atención específica en el aula.

\section{Análisis de la presentación de Yésica}

Tras analizar la presentación elaborada por Yésica en la segunda fase de la experiencia con las tres rúbricas de la tabla 1 obtuvimos los resultados de la tabla 7. En ella se observa que el valor del índice MNT es casi coincidente con el obtenido por el grupo A, al que pertenecía, el cual era mayor que el de los demás grupos. También hay en la presentación elaborada por Yésica un significativo anidamiento, debido a modos no textuales insertados en tablas, así como un uso nulo de elementos irrelevantes. Otro aspecto de interés es un número significativo de temas tratados (parámetro d del apartado MNT), como sucedía con el grupo A.

Tabla 7. Resultados numéricos de la presentación de Yésica.

\begin{tabular}{|c|c|c|c|c|c|c|c|}
\hline \multicolumn{4}{|c|}{ MNT } & \multicolumn{2}{|c|}{ EI } & \multicolumn{2}{|c|}{ CRI } \\
\hline Fotos o dibujos & 3 & a & 76 & PTI & 426 & PCRI & 191 \\
\hline Gráficas & 13 & $\mathrm{~b}$ & 6 & NCUM & 76 & NCUM & 76 \\
\hline Tablas & 30 & $\mathrm{c}$ & 0 & EI & 5,05 & CRI & 2,51 \\
\hline Listas & 13 & $\mathrm{~d}$ & 14 & Anidado & $15(20 \%)$ & & \\
\hline Diagramas & 11 & MNT & 96 & Original & $64(84 \%)$ & & \\
\hline Ecuaciones & 6 & & & & & & \\
\hline TOTAL & 76 & & & & & & \\
\hline Irrelevante & 0 & & & & & & \\
\hline
\end{tabular}

Sin embargo, aunque el número total de modos no textuales de representación de Yésica sea similar al del grupo A, no lo es del mismo tipo. En el grupo A, como ya se indicó, los elementos más habituales eran fotos y dibujos, a menudo cortados y pegados de Internet, por lo que la originalidad era baja (20\%), mientras que la presentación de Yésica tiene un uso variado de todos los modos no textuales de representación, incluyendo a los ausentes en todos 
los grupos del estudio inicial (ecuaciones y gráficas). Además, en el producto de Yésica hay párrafos de texto, ausentes en los productos del estudio inicial, y una originalidad destacable $(84 \%)$.

En cuanto al índice EI, el valor de Yésica es superior al del grupo al que pertenecía durante el estudio inicial y muy parecido al del grupo D (el que había obtenido mejor valor en el estudio previo), lo cual indica una mejora de la integración de los modos no textuales de representación de conocimiento. Esto se debe a la ausencia total de modos no textuales irrelevantes y al extenso uso de modos no textuales originales y anidados simultáneamente, características que raramente se daban al mismo tiempo en el estudio inicial. Finalmente, cabe indicar que el valor de CRI es superior al de todos los grupos del estudio inicial, lo cual muestra que es capaz de emplear modos no textuales de representación con una mayor capacidad de resumir información.

Podemos decir entonces que Yésica conserva aspectos característicos del estilo de su grupo, como el uso de numerosas tablas (cuyo número aumenta), pero mejorando aspectos importantes, como una originalidad mucho mayor. Por otra parte, quizás debido a la dificultad de hacer una exposición en solitario, Yésica incluye en su presentación algunos párrafos de texto, los cuales pueden ser expuestos tal cual, sin necesidad de ser reinterpretados.

\section{Análisis de la percepción y comprensión de los participantes}

En lo relativo al $2^{\circ}$ objetivo de este estudio, para profundizar acerca de la percepción de los participantes, primero hemos examinado las motivaciones de Yésica durante el proceso de toma de decisiones acerca del diseño de su presentación. Con ese fin hemos recopilado ensayos elaborados por ella, cuyo análisis, en base a los aspectos señalados por Hulleman y Harackiewicz (2009) y a la noción de acción comunicativa (Habermas 1987), lo resumimos en la tabla 8 (en cursiva, lo escrito por ella).

Tabla 8. Tipos de motivación detectados en los ensayos de Yésica.

\begin{tabular}{|l|l|}
\hline \multicolumn{1}{|c|}{ TIPO } & \multicolumn{1}{|c|}{ EJEMPLO } \\
\hline $\begin{array}{l}\text { Interés intrínseco por el } \\
\text { tema }\end{array}$ & $\begin{array}{l}\text { Se han introducido en la presentación las ecuaciones y gráficas porque son necesarias para los } \\
\text { dendrogramas }\end{array}$ \\
\hline Utilidad & $\ldots$ los dendrogramas] se pueden adoptar en cualquier área de investigación \\
\hline Desarrollo de destreza & $\ldots$ he aprendido cómo se usan y construyen [los dendrogramas] \\
\hline Acción comunicativa & $\begin{array}{l}\text { Incluir en la presentación ecuaciones, gráficos, tablas, fotos, etc. les ha permitido [a los } \\
\text { alumnos asistentes a la exposición] entender el tema }\end{array}$ \\
\hline
\end{tabular}

Yésica da importancia a la comunicación con otras personas, es decir, a la búsqueda de entendimiento y comprensión. Además del ejemplo de la tabla, hay otras frases en los ensayos de Yésica relevantes para el desarrollo de destrezas comunicativas, como "Elaborar la exposición de un tema permite que otras personas conozcan sobre el tema desarrollado". Igualmente nos parece relevante la importancia que le otorga a la ayuda del profesor, ya que también escribe: "gracias a la ayuda del profesor se ha podido terminar el desarrollo de la presentación de manera que fuese útil a los estudiantes en el momento de exponer el tema".

Esta información se contrasta con el registro anecdótico elaborado por el profesor; la interpretación que hacemos del mismo, con los mismos criterios que en la tabla 8 , se resume en la tabla 9 y apunta en la misma dirección: junto con los aspectos relacionados con el aprendizaje personal, se pone de manifiesto la orientación comunicativa dada por la alumna a sus acciones. También existe un interés por el éxito, por ejemplo cuando Yésica pregunta 
cómo se iba a puntuar la exposición, lo cual es plenamente lógico y comprensible, pero se relacionaría con lo que Habermas (1987) llama integración sistémica de las acciones.

Tabla 9. Tipos de motivación de Yésica registrados por el profesor.

\begin{tabular}{|l|l|}
\hline \multicolumn{1}{|c|}{ TIPO } & \multicolumn{1}{|c|}{ EJEMPLO } \\
\hline $\begin{array}{l}\text { Expectativas de éxito } \\
\text { escolar }\end{array}$ & Yésica preguntó cómo se iba a calcular la puntuación de la presentación \\
\hline $\begin{array}{l}\text { Interés intrínseco por el } \\
\text { tema }\end{array}$ & $\begin{array}{l}\text { Después de explicarle que los dendrogramas se pueden hacer en base a correlaciones, Yésica } \\
\text { recordó el significado y el cálculo de las correlaciones }\end{array}$ \\
\hline Utilidad & $\begin{array}{l}\text { Yésica preguntó por la relación de las gráficas y cálculos que hacemos con otros que bizo en } \\
\text { otros módulos formativos }\end{array}$ \\
\hline Desarrollo de destreza & Yésica preguntó que variables se usan en la gráfica final, las originales o las escaladas \\
\hline Acción comunicativa & Yésica les preguntó varias veces a los asistentes a su exposición si querían hacer preguntas \\
\hline
\end{tabular}

El último aspecto que hemos analizado es si el alumnado asistente a las exposiciones de Yésica adquiere una comprensión adecuada de los dendrogramas, así como conocer su percepción acerca de la pertinencia de los modos no textuales de representación empleados. En la tabla 10 se agrupan en categorías los resultados de la caracterización, iterativa y negociada entre los autores, de las respuestas de los estudiantes a las preguntas de la tabla 2.

Casi la totalidad de los asistentes comprenden la utilidad fundamental de los dendrogramas y son capaces de enumerar los pasos de su elaboración, pero algunos detalles importantes, como su naturaleza jerárquica o su forma de árbol solo son mencionados por algunos. Además, casi la mitad no presentan nuevos ejemplos de situaciones en las que se podrían emplear los dendrogramas, lo cual consideramos un indicio de que, para un número importante de estudiantes, el aprendizaje no es plenamente funcional.

En lo que respecta a la percepción de los asistentes referida al uso de modos no textuales de representación de conocimiento, es reseñable que todos manifiestan que su inclusión en la presentación les parece útil. Algunas de las razones que dan para ello son poco precisas ("para comprender mejor"), pero otras son más concretas ("para relacionar variables" o "para mostrar los pasos"); y otras se relacionan más con la respuesta y la atención de la audiencia que con la lógica de los contenidos ("para mantener la atención" o "para organizar de forma intuitiva").

\section{Conclusiones e implicaciones educativas}

En general, los resultados del estudio inicial muestran un importante uso de modos no textuales de representación de conocimiento científico por parte del alumnado. Estos modos no textuales de representación están aceptablemente integrados, siendo relevante el significativo número de casos en los que unos se anidan en otros, $\mathrm{y}$, además, muy pocos pueden considerarse irrelevantes. Sin embargo, hay una escasa capacidad de resumir información y, en algunas presentaciones, hay una escasa originalidad. Es interesante que los distintos grupos tienen diferentes estilos en sus presentaciones, lo cual provoca que los valores de las distintas dimensiones de la tabla 1 sean independientes. 
Tabla 10. Comprensión y percepción del alumnado asistente a la exposición $(\mathrm{n}=30)$.

\begin{tabular}{|c|c|c|}
\hline COMPRENSIÓN DE LOS DENDROGRAMAS & $\mathbf{n}$ & $\%$ \\
\hline Valora que agrupan objetos o variables de acuerdo a su similitud & 29 & 97 \\
\hline Menciona que tienen forma de árbol & 10 & 33 \\
\hline Menciona que son una técnica de análisis jerárquico & 6 & 20 \\
\hline Enumera los pasos de su elaboración & 28 & 93 \\
\hline Es capaz de poner nuevos ejemplos para su uso & 17 & 57 \\
\hline Diferencia entre variables manifiestas y fundamentales & 5 & 17 \\
\hline \multicolumn{3}{|l|}{$\begin{array}{l}\text { VALORACIÓN DE LA UTILIDAD DE MODOS NO TEXTUALES DE } \\
\text { REPRESENTACIÓN }\end{array}$} \\
\hline Considera que los modos no textuales de representación tienen alguna utilidad & 30 & 100 \\
\hline -Para facilitar poner ejemplos & 1 & 3 \\
\hline -Para mostrar pasos & 7 & 23 \\
\hline -Para comprender mejor & 14 & 47 \\
\hline -Para relacionar variables & 8 & 27 \\
\hline -Para mantener la atención & 1 & 3 \\
\hline -Para organizar de forma intuitiva & 2 & 7 \\
\hline
\end{tabular}

En el posterior estudio de caso, Yésica mejora el uso de modos no textuales de representación, en especial su originalidad y su capacidad de resumir información, siendo aumentada esta última en parte debido a la mayor inclusión de ecuaciones y gráficas. Esto se relaciona con la distinta naturaleza de los contenidos expuestos en la fase posterior con respecto a la inicial, dada la naturaleza abstracta y matemática de los dendrogramas. En las presentaciones hechas durante el estudio inicial, los temas a tratar estaban relacionados con la seguridad en los laboratorios, y los estudiantes usaban modos no textuales de representación que resumen menos información pero que interaccionan de forma eficaz con la información que dan, usando fundamentalmente fotos o dibujos en tablas o bien listas de normas. La naturaleza de los contenidos es, pues, decisiva en la calidad de los modos no textuales empleados. Es destacable que Yésica, aunque mejora los puntos que hemos indicado, conserva el estilo general de su grupo de la fase inicial, presentando la información en tablas. Además, su presentación no tiene errores e incluye ejemplos, lo cual nos informa de que logró un aprendizaje significativo y funcional acerca de los contenidos relacionados.

El análisis de su percepción muestra, además, que la alumna es capaz de valorar mejoras a nivel personal de desarrollo de destrezas o de éxito escolar, pero también percibe avances en términos comunicativos de hacer su exposición más inteligible para ayudar a sus compañeros a comprender mejor. Es decir, valora su progreso en términos comunicativos (Habermas 1987), orientándolo a la mejora común, buscando el diálogo y la comprensión mutua. En lo que a esto se refiere, es relevante la valoración positiva de Yésica de la ayuda del profesor, relacionándola con que le facilitase que la presentación elaborada sea útil a los asistentes a la exposición. Es decir, el papel del docente es percibido por la alumna como importante. La asistencia del profesor es para ella, pues, una ayuda para ayudar a comprender. Existen diferencias entre los resultados de distintas estrategias docentes para transferir responsabilidad a los estudiantes (Crujeiras y Jiménez 2018) y pensamos que se ha puesto en evidencia que ayudar a ser capaz de exponer de forma efectiva cosas a otros puede ser una forma de aumentar la eficacia de la enseñanza concebida como andamiaje, es decir, como la cesión de progresiva responsabilidad al alumnado. 
La mejora en el aumento de las destrezas de uso de modos no textuales de representación de conocimiento científico también se ha documentado en otros estudios (Reigosa 2010) y es positiva en términos de alfabetización científica (Prain y Hand 2016), y esto se refuerza aquí con un enfoque comunicativo, que permite un empoderamiento y una emancipación no solo individuales sino también compartidos, al ser capaces los estudiantes de manejar y comunicar el conocimiento con seguridad. Al progresar en la capacidad de expresarse usando el lenguaje de la ciencia, no se beneficia solo cada alumna o alumno individualmente, sino además todos aquellos con los que interactúa.

Esta interpretación se ve refrendada por la percepción del alumnado asistente a la exposición de Yésica, ya que, si bien no todos son capaces de alcanzar una comprensión completa y funcional de los contenidos expuestos, sí que en su totalidad adquieren una comprensión básica y además consideran útiles los modos no textuales de representación empleados.

En definitiva, pensamos que, esta experiencia de investigación-acción aporta resultados que ponen de manifiesto que es posible progresar en la alfabetización científica del alumnado incidiendo en el uso de modos no textuales de representación del conocimiento. Consideramos esto especialmente importante con alumnado adulto, como el de este estudio, y está en línea con el estudio de Spektor-Levy et al. (2009), el cual muestra que la instrucción marca la diferencia en las destrezas de comunicar conocimiento científico de los estudiantes, a lo cual vemos que no es ajeno el uso de modos no textuales empleados con tal finalidad. En otro estudio, Gebre y Polman (2016) muestran que para mejorar el uso de representaciones más abstractas por parte de los estudiantes es clave la ayuda del docente. Debido a la gran variedad de modos no textuales de representación y a la constatación de que distintos contenidos propician el uso de distintos modos de representación, consideramos que sería conveniente que los estudiantes tuvieran la oportunidad de enfrentarse a diversos tipos de contextos que implicasen temáticas de distinta naturaleza, así como de realizar exposiciones individuales y colectivas, lo cual favorecería que, de forma reflexiva y dialogada, tomasen conciencia de lo que las imágenes y otros modos no textuales de representación comunican a la audiencia. Esto debería trabajarse intencionalmente en el aula, sin suponer que sea un aprendizaje que se adquiere espontáneamente; además, sería de interés investigarlo también en otros niveles educativos.

\section{Agradecimientos}

Paloma Blanco Anaya agradece la financiación por FEDER/Ministerio de Ciencia, Innovación y Universidades Agencia Estatal de Investigación/ Proyecto ESPIGA, referencia PGC2018-096581-B-C22

\section{Referencias bibliográficas}

Crujeiras, B., Jiménez, M.P. (2018) Influencia de distintas estrategias de andamiaje para promover la participación del alumnado de secundaria en las prácticas científicas. Enseñanza de las Ciencias 36(2), 23-42. https://doi.org/10.5565/rev/ensciencias.2241

Cunningham, A. (1988) Getting the same right: some plain words on the identity and invention of science. Studies in History and Phylosophy of Science 19(3), 365-389. https:/ / doi.org/10.1016/0039-3681(88)90005-2

De Arriba, J.D. (2007) Educar para participar en educación de personas adultas mediante una alfabetización científica con orientación CTS. Revista Iberoamericana de Educación 44(2), 1 7.

Flick, U. (2004) Introducción a la investigación cualitativa. Madrid: Morata. 
Gebre, E.H., Polman, J.L. (2016) Developing young adults' representational competence through infographic-based science news reporting. International Journal of Science Education 38(18), 2667-2687. https://doi.org/10.1080/09500693.2016.1258129

Gil, D., Carrascosa, J., Martínez, F. (2000) Una disciplina emergente y un campo específico de investigación. En J. Perales y P. Cañal (Eds.), Didáctica de las ciencias experimentales. (pp. 1134). Alcoy: Marfil.

Giroux, H.A. (1990) Los profesores como intelectuales. Hacia una pedagogía crítica del aprendizaje. Barcelona: Paidós.

Glaser, B., Strauss, A. (1967) The discovery of grounded theory: strategies for qualitative research. Nueva York: Aldine.

Habermas, J. (1987) Teoría de la acción comunicativa I: Racionalidad de acción y racionalización social. Madrid: Taurus.

Hulleman, C.S., Harackiewicz, J.M. (2009) Promoting interest and performance in high school science clases. Science 326, 1410-1412. https://doi.org/10.1126/science.1177067

Kvale, S. (1996) Interview Views: An Introduction to Qualitative Research Interviewing. Thousand Oaks: Sage Publications.

Kemmis, S., Mc'Taggart, R. (1988) Como planificar la investigación-acción. Barcelona: Laertes.

Latour, B., Woolgar, S. (1967) La vida en el laboratorio. Madrid: Alianza.

Lemke, J. (1997) Aprender a hablar ciencia: lenguaje, aprendizaje y valores. Barcelona: Paidós.

Lundsford, E., Melear, C.T., Roth, W.-M., Perkins, M., Hickok, L.G. (2007) Proliferation of inscriptions and transformations among preservice science teachers engaged in authentic science. Journal of Research in Science Teaching 44(4), 538-564. https://doi.org/10.1002/tea.20160

McDermott, M., Hand, B. (2013) The impact of embedding multiple modes of representation within writing tasks on high school students' chemistry understanding. Instructional Science 41, 217-246. https:/ / doi.org/10.1007/s11251-012-9225-6

McDermott, M., Hand, B. (2016) Modeling scientific communication with multimodal writing tasks: impact on students at different grade levels. En B. Hand, M. McDermott y V. Prain (Eds.), Using multimodal representations to support learning in the science classroom. (pp. 183-211). Londres: Springer.

McKernan, J. (1999) Investigación-acción y curriculum. Madrid: Morata.

Moje, E. (2007) Developing socially just subject-matter instruction: A review of the literature on disciplinary literacy learning. Review of Research in Education 31, 1-44. https://doi.org/10.3102/0091732X07300046001

OCDE (2016) How does PISA assess science literacy?. PISA in Focus 66. Paris: OECD Publishing. https://doi.org/10.1787/5jln4nfnqt7l-en

Perales, J. (2006) Uso (y abuso) de la imagen en la enseñanza de la ciencia. Enseñanza de las Ciencias 24(1), 13-30.

Prain, V, Hand, B. (2016) Learning science through learning to use its languages. En B. Hand, M. McDermott y V. Prain (Eds.), Using multimodal representations to support learning in the science classroom (pp. 1-10). Londres: Springer.

Ramis, G., García. M.C. (2001) Quimiometría. Madrid: Síntesis. 
Reigosa, C. (2010) Un estudio de caso sobre la influencia del uso de una herramienta informática sobre las destrezas de comunicación científico-técnicas y el aprendizaje conceptual. Revista Electrónica de Enseñanza de las Ciencias 9(1), 186-198.

Salvá, F.A., Latorre, A. (1998) La alfabetización científica de personas adultas: un enfoque comunicativo. Enseñanza de las ciencias 16(2), 251-260.

Sárrate, M.L. Pérez, M.V. (2005) Educación de personas adultas. Situación actual y propuestas de futuro. Revista de Educación 336, 41-57. https://doi.org/10.4438/1988-592X-00348082-RE

Spektor-Levy, O., Eylon, B. S., Scherz, Z. (2009) Teaching scientific communication skills in science studies: Does it make a difference?. International journal of science and mathematics education 7(5), 875-903. https:// doi.org/10.1007/s10763-009-9150-6

Vázquez, B, Jiménez, R. y Mellado, V. (2019) El contenido didáctico del contenido (CDC) de una profesora de ciencias: reflexión y acción como facilitadores del aprendizaje. Enseñanza de las Ciencias 37(1), 25-53. https://doi.org/10.5565/rev/ensciencias.2550

Vygotski, L.S. (2010) Pensamiento y lenguaje. Segunda edición. Barcelona: Paidós. (Original de 1934).

Wertsch, J.V. y Rupert, L.J. (1993) The Authority of Cultural Tools in a Sociocultural Approach to Mediated Agency. Cognition and Instruction, $11(3$ \& 4), 227-239. https://doi.org/10.1080/07370008.1993.9649022

Yin, R. K. (2003) Case study research: design and methods (3 ed.). Londres: SAGE Publications. 